\title{
Cost analysis of a system of ad hoc theatre sessions for the management of delayed trauma cases
}

\author{
Oreste Basso
}

Received: 22 October 2007/Accepted: 15 December 2008/Published online: 31 January 2009

(c) Springer-Verlag 2009

\begin{abstract}
Background Reducing hospital stay optimizes bed capacity. Shortage of operating time can cause some patients to have their treatment and discharge home delayed. Extra operating sessions could help in reducing such a delay. We performed a feasibility study for a simulated model of trauma lists, implemented ad- hoc to reduce time to surgery.

Materials and methods Two hundred thirty-five consecutive trauma admissions were audited. The time required to deliver surgical treatment was recorded. Patients waiting for their operation more than $48 \mathrm{~h}$ from admission were allocated into a simulated system of ad hoc trauma lists, using a realistic decision-making process. The potential to reduce time-to-operation was assessed and the number of saved bed occupancy days was calculated. A cost analysis was also performed.

Results Surgical treatment was delivered within $48 \mathrm{~h}$ in $193(85 \%)$ patients, while $32(15 \%)$ patients waited a mean of 3.8 days (3-7), because of insufficient time. To operate on these patients earlier, additional lists would have cost $£ 38$, 703 , reducing the time to surgery to 1 day (0-2). This would have saved 86 days of bed occupancy, representing a savings of $£ 17,200$. Restricting the use of extra lists to the elderly patients in the cohort would have required only 11 extra lists and reduced waiting from $3(3-4)$ to 1 days (0-2), for a cost of $£ 22,407$. Elderly patients' lists would have had space left to treat additional seven younger patients, with a total saving of 51 bed occupancy days, corresponding to $£ 10,200$.
\end{abstract}

O. Basso $(\square)$

Department of Trauma and Orthopaedics, Newham University

Hospital, Glen Rd, London E13 8SL, UK

e-mail: Oreste.basso@newhamhealth.nhs.uk;

Oreste_basso@libero.it
Conclusions The system of ad hoc trauma lists is easy to organize and it appears to impact significantly on patients' discharge and bed capacity. Direct costs to the health service are contained, as they are partially compensated by the improvement in beds availability.

Keywords Cost analysis - Audit - Trauma . Fracture neck of femur $\cdot$ Bed management

\section{Introduction}

Achieving an optimal balance between clinical excellence and budget containment is a primary target of modern healthcare services. Reducing patients' in-hospital length of stay contributes to optimize bed capacity with potential financial returns. Sometimes the number of patients with traumatic conditions awaiting operation exceeds the capacity of operating theatres, resulting in a delay in the discharge home. Extra operating sessions could help to contain such a problem. To our knowledge, the financial implications of this option have not been assessed before. This is an audit of the surgical activity of a typical district hospital of the British National Health Service, assessing the potential costs and benefits of a system of "ad hoc" theater sessions, implemented to reduce time to surgery for patients admitted with trauma. We focused on direct costs to the health service, improvements in bed capacity and the impact on patients.

\section{Materials and methods}

All consecutive emergency orthopaedic admission, presenting to Luton and Dunstable District Hospital in a 
13-week period were audited prospectively. The epidemiology of fractures (Table 1) and the time required to deliver surgical treatment were recorded by the author. Demographic data, date of admission, planned operation and the date of operation were entered into the trauma database by the trauma coordinator, an experienced nurse whose exclusive task is to follow-up all trauma patients and optimize the pathway from admission to discharge. Following anaesthetic clearance, patients were operated on as soon as possible.

Planning of surgical activity

At our hospital, a Monday to Saturday session is available for a consultant-staffed trauma list. In the British National
Table 1 Epidemiology of all fractures (ten excluded cases not represented)
Operating times refer to 'skin to skin' (start to end of anaesthetics, in case of manipulation)

$I M$ intra medullary, MUA manipulation under anaesthesia, $K$-wiring Kirschner wiring

\begin{tabular}{|c|c|c|c|}
\hline Fractures & $N$ & Operation & $\begin{array}{l}\text { Operating time } \\
\text { allowance }(\mathrm{min})\end{array}$ \\
\hline Subcapital femoral fracture & 25 & Hemiarthroplasty & 60 \\
\hline Subcapital femoral fracture & 9 & Cannulated screws & 60 \\
\hline Intertrochanteric fracture & 17 & Sliding screw & 60 \\
\hline Femur & 5 & IM nailing & 90 \\
\hline Femur (12 years old) & 1 & MUA + cast & 30 \\
\hline Supracondylar femur & 1 & Nailing & 90 \\
\hline Supracondylar femur & 1 & Internal fixation & 90 \\
\hline Periprosthetic (hip arthroplasty) & 1 & Revision stem & 90 \\
\hline Tibia plateau & 6 & Internal fixation & 90 \\
\hline Tibia plateau & 1 & Circular frame & 90 \\
\hline Tibial diaphysis & 2 & MUA + cast & 30 \\
\hline Tibial diaphysis & 3 & Unreamed nailing & 90 \\
\hline Tibial diaphysis & 1 & External fixation & 60 \\
\hline Tibial diaphysis & 1 & Plating & 60 \\
\hline Distal tibia & 2 & Internal fixation & 90 \\
\hline Bimalleolar fracture & 17 & Internal fixation & 60 \\
\hline Distal radius & 30 & MUA + wiring & 60 \\
\hline Distal radius & 4 & Plating & 60 \\
\hline Shoulder dislocations & 3 & MUA & 30 \\
\hline Proximal humerus & 2 & Internal fixation & 90 \\
\hline Humeral diaphysis & 1 & Internal fixation & 90 \\
\hline Hand fractures/dislocation & 16 & MUA + K wiring & 60 \\
\hline Scaphoid fracture & 1 & Internal fixation & 60 \\
\hline Tendons injury & 6 & Repair & 60 \\
\hline Infected fixation device & 8 & Removal & 60 \\
\hline Other infections/hematoma & 18 & Drainage & 30 \\
\hline Wrist tendons laceration & 3 & Repair & 45 \\
\hline Rotator cuff rupture & 1 & Open repair & 60 \\
\hline Acute derangement of knee & 5 & Arthroscopy & 60 \\
\hline Dislocated hip prostheses & 5 & MUA & 30 \\
\hline Radius-ulna diaphyses & 7 & MUA & 30 \\
\hline Radius-ulna diaphyses & 4 & Internal fixation & 60 \\
\hline Elbow dislocations & 2 & MUA & 30 \\
\hline Radial head & 5 & Internal fixation & 60 \\
\hline Supracondylar humeral fracture & 2 & MUA + wiring & 60 \\
\hline Patella & 3 & Internal Fixation & 60 \\
\hline Arm laceration & 3 & Repair & 60 \\
\hline Foreign body & 3 & Removal & 60 \\
\hline Total & 225 & & \\
\hline
\end{tabular}


Health Service, a session is equivalent to $41 / 2 \mathrm{~h}$ actual work. During the week, the first patient on the trauma list is scheduled to arrive at the theater no later than $13.30 \mathrm{~h}$ and the last patient to leave for the recovery area no later than 18. On Saturdays, start and end times are 9 and 13.30. Trauma operating lists are updated daily during the trauma meetings, occurring every morning at 8.00 , Monday to Saturday, when the consultant on call for the previous $24 \mathrm{~h}$ reviews the cases admitted during his on take period. At the end of the meeting, the details of the new admissions, selected for surgery, are added to the trauma waiting list in chronological order of arrival. Patients are allocated a place on the first available trauma list, according to their order of admission. Specific issues, such as preoperative conditions, young or elderly age, severity of the injury are generally given priority. The number of cases to place on each operating list depends on the type and estimated duration of the planned operations. The total operating time for each trauma list is calculated by summing up the estimated operating times of each procedure (Table 1). A period of $30 \mathrm{~min}$ is added to allow for patient's changeover, which includes the time required to resuscitate and transfer to recovery area and then check in the following patient. In general, the estimated duration of each procedure is an excess approximation of the trend of the actual operating time, required to carry out surgery from 'knife-to-skin' until completed closure, as recorded in the operative register. The target of the trauma lists is for patients to receive their operation within $48 \mathrm{~h}$ from admission. Occasionally, planned delay can occur, due to the need to optimize preoperative condition or the requirement for specific instrumentation/expertise. To improve operating capacity, some patients are booked by the trauma coordinator on elective lists, following last-minute cancellations and on the emergency lists. During weekends, it is the responsibility of the on-call consultant to review and allocate cases to the Saturday morning trauma list and the emergency lists of Saturday and Sunday. During particularly busy times, the combined capacity of the trauma-emergency-elective lists system is exceeded and some patients will be scheduled to have their operation later than $48 \mathrm{~h}$. Also, it can happen that a patient, originally scheduled within $48 \mathrm{~h}$, breaches the original plan, because of an unexpected delay in completing surgery on an earlier case.

\section{The simulation}

For those patients who had their operation beyond $48 \mathrm{~h}$, we hypothesized that extra trauma lists could be organized ad hoc within $24 \mathrm{~h}$ from admission, using any available staff willing to undertake extra work for an additional payment.
The construction of the simulated extra lists followed the same principles used for the daily organization of the actual trauma work, according to a realistic decision-making process. Each simulated extra list was compiled during the week by the author, who:

- identified any patient on the trauma board, due to receive surgery later than $48 \mathrm{~h}$

- identified available extra theatre time and available offduty personnel within $24 \mathrm{~h}$

- assessed operating time (Table 1), assigning to each extra list the right number of cases.

The number of bed occupancy days that could have been saved, had the extra lists been implemented, was obtained by calculating the difference in days, between actual and simulated dates of operation (Table 2). An analysis of the costs of the simulated extra lists was performed by the finance department (Table 3). The cumulative number of notional saved bed- days was multiplied by the figure of $£ 200$, which is an average estimate by the hospital finance department of ward costs and overheads over a 10-day period of hospital stay.

\section{Results}

A total of 235 consecutive trauma admissions requiring surgical management were recorded. The mean age for all patients was 51.3 (4-106). The M:F ratio was 125:110. Thirty-one patients were less than 16 years old (mean 11.3 , range 4-15). Eighty-eight patients were 65 or older (mean 80.3, range 66-106). There were 54 fractures of neck of femur (NOF), representing 23\% of the total (Table 1).

Exclusions Ten of 235 patients were excluded. Nine of ten exclusions presented conditions causing delay in scheduling surgery other than lack of operating time, such as the requirement for specific instrumentation/expertise (five cases of spinal stabilization and one complex knee ligaments injury) and the need to optimize preoperative status (three cases). In one case, information available was inadequate.

Analysis of the hospital's surgical registers revealed that of the 225 patients included in the study (Table 1), 108 were operated on 58 regular trauma lists, 109 on emergency lists, of which 25 cases during weekends (including bank holiday Monday, May 1) and the rest on elective lists. One-hundred and ninety-three of these 225 $(85 \%)$ patients (group 1) received their operation within $48 \mathrm{~h}$ from admission. The remaining 32 (Table 2) patients (group 2) had a mean time to operation of 3.8 days (3-7), for reasons related to the lack of operating time. Again, this was despite the use of spare room on elective lists 
Table 2 Group 2-Comparison of actual and simulated surgical activity

\begin{tabular}{|c|c|c|c|c|c|c|c|}
\hline Pts & Age & Planned operation & $\begin{array}{l}\text { Admission } \\
\text { date }\end{array}$ & $\begin{array}{l}\text { Actual lists } \\
\text { date }\end{array}$ & $\begin{array}{l}\text { Days to } \\
\text { surgery }\end{array}$ & $\begin{array}{l}\text { Simulated } \\
\text { lists }\end{array}$ & $\begin{array}{l}\text { Notional } \\
\text { saved days }\end{array}$ \\
\hline 1 & 40 & Plating tibial plateau & 29-March & 03-April (T) & 5 & (31 March) & 3 \\
\hline 2 & 49 & Evac. calf hematoma & 30-March & 04-April (T) & 5 & & 4 \\
\hline 3 & 49 & External fixation tibia & 30-March & 04-April (T) & 5 & & 4 \\
\hline 4 & 22 & Plating tibial plateau & 12-April & 15-April (T) & 3 & (13 April) & 2 \\
\hline 5 & 73 & Rem. metalwork (Ankle) & 18-April & 21-April (E) & 3 & (19 April) & 2 \\
\hline 6 & 79 & Sliding hip screw & 21-April & 24-April (T) & 3 & (23 April) & 1 \\
\hline 7 & 84 & Sliding hip screw & 23-April & 26-April (E) & 3 & & 3 \\
\hline 8 & 83 & Manipulation of shoulder & 24-April & 27-April (T) & 3 & (26 April) & 1 \\
\hline 9 & 95 & Sliding hip screw & 25-April & 28-April (E) & 3 & & 2 \\
\hline 10 & 22 & Wiring thumb M. carpal & 29-April & 02-May (T) & 3 & (30 April) & 2 \\
\hline 11 & 23 & Plating radial head & 01-May & 04-May (T) & 3 & (2 May) & 2 \\
\hline 12 & 20 & Plating bimalleolar \# & 03-May & 06-May (T) & 3 & (4 May) & 2 \\
\hline 13 & 94 & Sliding hip screw & 03-May & 06-May (T) & 3 & & 2 \\
\hline 14 & 42 & Plating distal radius & 03-May & 07-May (E) & 4 & & 3 \\
\hline 15 & 83 & Hemiarthroplasty hip & 05-May & 08-May (T) & 3 & (6 May) & 2 \\
\hline 16 & 16 & Nailing of tibia & 05-May & 09-May (T) & 4 & & 3 \\
\hline 17 & 68 & Plating tibial plateau & 07-May & 12-May (T) & 5 & (8 May) & 4 \\
\hline 18 & 89 & Manipulation tibia & 09-May & 13-May (T) & 4 & (10 May) & 3 \\
\hline 19 & 57 & I and D thigh abscess & 09-May & 13-May (E) & 4 & & 3 \\
\hline 20 & 87 & Manipulation distal radius & 10-May & 13-May (E) & 3 & (11 May) & 2 \\
\hline 21 & 37 & Removal external fixator & 11-May & 15-May (T) & 4 & & 4 \\
\hline 22 & 48 & Plating shaft of tibia & 19-May & 23-May (T) & 4 & (20 May) & 3 \\
\hline 23 & 64 & Periprosthetic no. (revision) & 29-May & 05-June (T) & 7 & (1 June) & 4 \\
\hline 24 & 30 & Plating radius-ulna shafts & 30-May & 03-June (T) & 4 & (31 May) & 3 \\
\hline 25 & 59 & Plating proximal humerus & 04-June & 09-June (T) & 5 & (5 June) & 4 \\
\hline 26 & 12 & Manipulation radial head & 05-June & 09-June (El) & 4 & & 4 \\
\hline 27 & 66 & Plating bimalleolar fract. & 06-June & 09-June (E) & 3 & (8 June) & 1 \\
\hline 28 & 22 & Removal foreign body & 08-June & 12-June (T) & 4 & & 4 \\
\hline 29 & 82 & Hemiarthroplasty hip & 16-June & 19-June (T) & 3 & (18 June) & 1 \\
\hline 30 & 64 & Plating bimalleolar fract. & 18-June & 22-June (T) & 4 & & 4 \\
\hline 31 & 91 & Nailing distal femur & 21-June & 24-June (T) & 3 & (22 June) & 2 \\
\hline 32 & 82 & Plating bimalleolar fract. & 21-June & 24-June (E) & 3 & & 2 \\
\hline
\end{tabular}

Patients 1, 7, and 23 had originally been allocated to a regular trauma list within $48 \mathrm{~h}$

$T$ trauma list, $E$ emergency list, $E l$ elective list

and evenings/weekends. Patients 1,7 and 23 (Table 2) had originally been allocated on a trauma list within $48 \mathrm{~h}$ from admission; however, operating time became insufficient because of unexpected delay in completing surgery on earlier cases. The mean age of group 2 cohort was 57.3 years (12-95). Six of the 32 delayed patients were cases of fractured NOF. Each of these NOF patients was operated on the third day from admission (Table 2). Fourteen of the 32 delayed patients were 65 or older (mean 82.6; range 66-95). The mean delay in this subgroup was 3.1 days (3-4).
Analysis of costs

A cost analysis is presented in Table 4. Implementing the system of extra lists for all 32 'delayed' patients (Table 2) would have reduced the number of days to operation from 3.8 (3-7) to 1 day (0-2), saving 86 days of bed occupancy. Implementing the extra lists, to treat just the 14 elderly patients in group 2, would have required only 11 lists, which in our specific case-series, would have allowed operating on seven additional younger patients. The time to operation for these 'elderly' extra lists (including the 
Table 3 Breakdown of costs per list (2006 figures): hours to be worked outside the normal programmed activities

\begin{tabular}{lr}
\hline Currency & \multicolumn{1}{c}{$£$} \\
\hline Materials and drugs (estimated average) & 762 \\
Staff costs & \\
Anaesthetist & 580 \\
Surgeon & 500 \\
Band 5 technician & 68 \\
Band 5 scrub nurse & 70 \\
Band 6 scrub nurse & 87 \\
Band 5 recovery staff & 70 \\
Total costs per list & 2,037 \\
\hline
\end{tabular}

younger patients squeezed in) would be reduced from 3 days (3-4) to 1 day (0-2). The total number of bed occupancy days potentially saved in this latter hypothesis was 51 (Tables 2, 4).

\section{Discussion}

This article reports the details of the trauma activity of a typical district hospital of the British National Health Service in a specific period of time. Epidemiological data are presented which can be useful to both researchers and health care providers. The problem of the delay in time to surgery, affecting some trauma patients, is dealt with by testing the feasibility of organizing a system of ad hoc trauma lists and observing both the effects that such a system is likely to produce on the time to operation and its costs and gains. The hypothesis that such a system is easy to organize and affordable is tested.

Can the implementation of extra lists reduce waiting time? In our setting, 32 of $225(15 \%)$ patients, having surgical management delayed for 3.8 days, could have been treated within $48 \mathrm{~h}$. It is reasonable to believe that, as a result of an earlier operation, they would have been discharged home earlier. It is generally accepted that elderly patients deserve even more a timely surgical treatment [1]. In our case, the extra lists would have reduced our elderly cohort's wait from 3.1 (3-4) to 1.1 days (0-2), which is less than half the actual figure. Is it worthwhile spending extra resources to achieve this target for all patients, regardless of age? From the patient's perspective, anyone would like to leave hospital earlier. From an ethical viewpoint, apart from the need to treat elderly patients promptly, everybody deserves the benefits of reduced pain and suffering, prevention of expensive to treat illness and shorter sick leave. However, the health care provider perspective may be different. What are the actual incremental costs of each extra list? Our calculations are essentially a business-case and provide sample-specific indications on costs, which can help other trusts plan their individual cases. Are there any hidden gains that could compensate for the costs incurred? Discharging patients earlier saves bed occupancy days, which makes a number of beds available to be used to boost elective activity. This can in turn generate resources that at least partially compensate the costs of the ad hoc lists system.

A limitation of our analysis is that typical of a business case: it represents a hypothetical exercise. The figures for bed-days saving reported are notional and not actual. In order to make notional savings real, administrators need to decide first whether the 86 bed-days, identified as potentially salvageable are enough to justify closing beds. The freed beds could then be occupied by elective patients and only then acquire an economical value. However, our study strongly suggests that the ad hoc lists system has a significant potential to improve bed capacity.

The figure of $£ 200$, indicated as the cost of each bed occupancy day by the trust may be smaller than the actual costs incurred. Calculations are in fact based on an average per diem forfeit figure. In reality, costs during the earlier stages of hospital stay can be higher [2] than the costs incurred later on in the stay. The delay assessed in our study occurred during the pre-operative period, which typically requires more intensive treatment and a larger direct nursing time [3]. It is therefore likely that the actual costs of each bed day in our study were in fact higher than the figures indicated by the trust. Another limitation of our study is the lack of a sensitivity analysis on the costs of bed occupancy. It is reasonable to believe, however, that if any variation is to be considered of the cost of a bed-occupancy day that can only be in terms of higher actual costs. This would then reinforce the contribution that this specific figure gives to the cost-benefit equation.

Table 4 Estimated costs and savings of extra lists

\begin{tabular}{lccc}
\hline Incremental costs & Bed-occupancy savings & \\
\hline All 32 delayed patients: & 14 elderly patients $(+7$ younger): & 32 patients: 86 days & 14 elderly (+7 younger): \\
19 lists required & 11 lists required & & 51 days \\
$£ 38,703$ & $£ 22,407$ & $£ 17,200$ & $£ 10,200$ \\
\hline
\end{tabular}

An alternative system where only elderly patients would be treated is also considered. In our simulation, this alternative option would have room left over, accommodating seven younger patients 
Our findings are based on the observation of a specific period of time within a specific geographical area. They are not therefore necessarily generalizable to other trusts and may be sensitive to seasonal variations. It is advisable that trusts conduct similar audits locally, to optimize the external validity.

Conflict of interest statement The author declares that he has no conflict of interest.

\section{References}

1. Hollingworth W, Todd C, Parker M, Roberts JA, Williams R (1993) Cost analysis of early discharge after hip fracture. Br Med J 307:906-909

2. Parker MJ, Myles JW, Anand JK, Drewett R (1992) Cost- benefit analysis of hip fracture treatment. J Bone Joint Surg (B) 74-B:261-264

3. Reid N, Robinson G, Todd C (1991) The quantity of nursing care on wards working 8-h and 12-h shifts. Int J Nurs Stud 28:47-54 